\title{
Full Sphere Axisymmetric Simulations of the Solar Dynamo
}

\author{
Dibyendu Nandy ${ }^{1}$, Piyali Chatterjee ${ }^{2}$ and Arnab Rai Choudhuri ${ }^{2}$ \\ ${ }^{1}$ Department of Physics, Montana State University, Bozeman, MT 59717, USA \\ email: nandi@mithra.physics.montana.edu \\ ${ }^{2}$ Department of Physics, Indian Institute of Science, Bangalore 560012, India
}

\begin{abstract}
We explore a full sphere (2D axisymmetric) kinematic solar dynamo model based on the Babcock-Leighton idea that the poloidal field is generated in the surface layers from the decay of tilted bipolar solar active regions. This model incorporates the helioseismically deduced solar rotation profile and an algorithm for buoyancy motivated from simulations of flux tube dynamics. A prescribed deep meridional circulation plays an important role in the advection of magnetic flux. We specifically address the parity issue and show that - contrary to some recent claims - the Babcock-Leighton dynamo can reproduce solar-like dipolar parity if certain reasonable conditions are satisfied in the solar interior, the most important requirement being that the poloidal field of the two hemispheres be efficiently coupled across the equator.
\end{abstract}

\section{Summary of Main Results}

In the last decade or so, simulations of the buoyant rise of magnetic flux tubes from the base of the solar convection zone (SCZ) have established that the strength of the strong sunspot forming toroidal magnetic fields must be of the order of $10^{5}$ Gauss $(\mathrm{G})$. Since the equipartition field strength in the SCZ is believed to be about $10^{4} \mathrm{G}$, the traditional $\alpha$-effect mechanism (for poloidal field production) involving small-scale helical turbulence would fail to act on much stronger flux tubes having field strengths $\sim 10^{5} \mathrm{G}$. Therefore other $\alpha$-effect mechanisms need to be considered for the production of the solar poloidal field. Amongst the alternatives, the Babcock-Leighton (BL) $\alpha$-effect has been explored in great detail in the recent past and has been found to reproduce many features of the solar cycle reasonably well (see Choudhuri et al. 1995; Nandy \& Choudhuri 2000, 2001, 2002; Nandy 2002, 2003 and references therein).

Recently however, Dikpati \& Gilman (2001) and Bonanno et al. (2002) claimed that the BL model may not reproduce the observed (odd or dipolar) parity of the magnetic fields (also manifested in the anti-symmetric distribution of the toroidal field across the solar equator). We explored this parity issue within the framework of our BL dynamo model (Nandy \& Choudhuri 2001, 2002) and found that the BL dynamo can reproduce the correct solar dipolar (odd) parity if the poloidal field of the two hemispheres is able to couple across the equator due to an enhanced diffusivity $\left(\sim 2.4 \times 10^{12} \mathrm{~cm}^{2} \mathrm{~s}^{-1}\right)$ in the SCZ (Chatterjee, Nandy \& Choudhuri 2004).

We show in Figure 1 a butterfly diagram for the toroidal field at the base of the SCZ superimposed on the radial field evolution contours. This butterfly diagram is constructed at the end of a 3000 year run which was initialized by a magnetic field distribution having quadrupolar parity (symmetric toroidal field across the equator). It is clear from the plot that the magnetic field distribution attains a solar-like dipolar parity with antisymmetric toroidal field across the equator. This model also reproduces the correct phase relationship between the poloidal and toroidal fields (polar field reversals at time of sunspot 


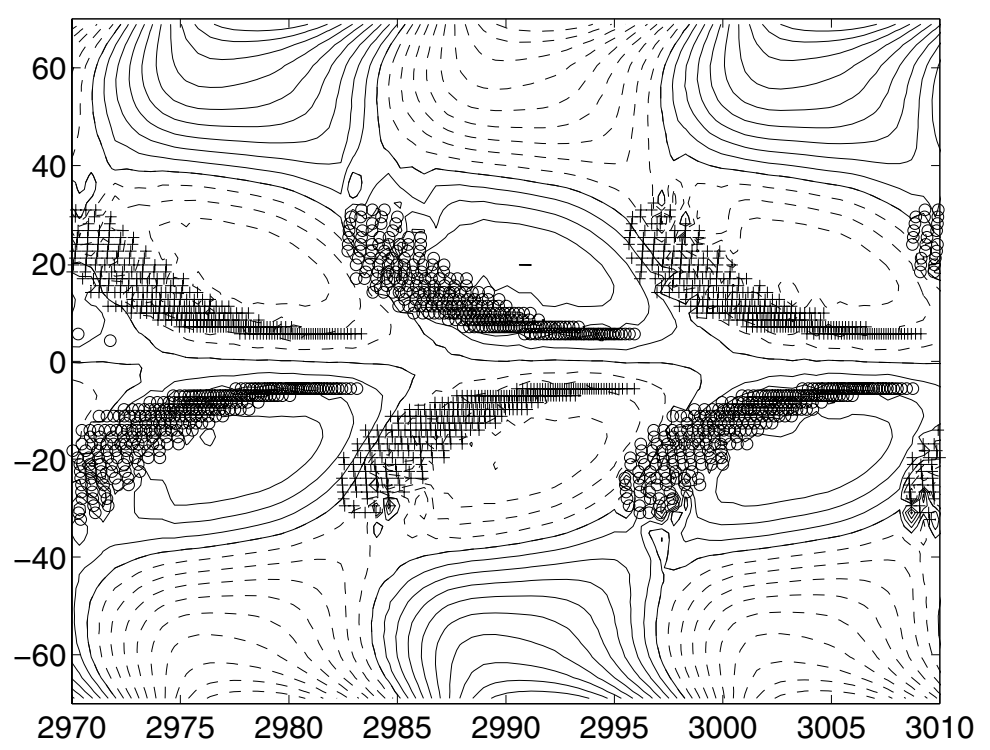

Figure 1. Theoretical butterfly diagram for buoyant sunspot eruptions (critical field for eruptions $=10^{5}$ Gauss) superimposed on the radial field evolution contours. Eruptions from an underlying positive toroidal field are denoted by ' + ' and from negative toroidal fields by 'o' signs respectively. Background shows the surface radial field evolution with time, positive radial field being denoted by solid and negative radial fields by dashed contours respectively. The $\mathrm{y}$-axis shows the latitude and $\mathrm{x}$-axis shows the time in years.

maxima and change of sign of the polar field from negative to positive when the underlying low-latitude toroidal field is positive) and works even when a quenching is used to mimic the feedback of the strong toroidal field on the deep meridional flow (with a peak surface meridional flow speed $\sim 22 \mathrm{~m} \mathrm{~s}^{-1}$ ). Our simulations for longer times, show that once the solution reaches a dipolar configuration, it stays in that solar-like parity state. In conclusion, we find that the BL mechanism for the solar dynamo can reproduce many of the well-known features of the solar cycle, including the correct parity, and remains a viable and promising model for the solar cycle.

For a detailed description of the BL dynamo mechanism, interested readers are referred to Nandy \& Choudhuri (2001, 2002), Nandy (2002, 2003) and Chatterjee, Nandy \& Choudhuri (2004). Dibyendu Nandy acknowledges financial support from NASA through Science Research \& Technology grant NAG5-11873.

\section{References}

Bonanno, A., Elstner, D, Rüdiger, G., \& Belvedere, G., 2002, A\&A, 390, 67

Chatterjee, P., Nandy, D., \& Choudhri, A. R. 2004, A\&A, 427, 1019

Choudhuri, A.R., Schüssler M., \& Dikpati M. 1995, A\&A, 303, L29

Dikpati, M., \& Gilman, P.A. 2001, ApJ, 559, 428

Nandy, D., 2002 Astrophysics \& Space Science, 282, 209

Nandy, D., 2003, ESA SP-517 (Proceedings of SOHO 12/GONG+ 2002), 123

Nandy, D., \& Choudhuri, A.R. 2000, J. Astrophys. Astron., 21, 381

Nandy, D., \& Choudhuri, A.R. 2001, ApJ, 551, 576

Nandy, D. \& Choudhuri, A.R., 2002, Science, 296, 1671 